\title{
Association between Helicobacter Pylori and Gastric Cancer
}

\author{
Brindeswari Kafle, ${ }^{1}$ Ramesh Singh Bhandari, ${ }^{2}$ Paleswan Joshi Lakhey, ${ }^{2}$ Pradeep Shrestha, ${ }^{1}$ Prem Khadka, \\ Shashi Sharma ${ }^{1}$ \\ 'Department of Medicine, ${ }^{2}$ Department of Surgery, Tribhuvan University Teaching Hospital, Kathmandu, Nepal.
}

Introduction: A casual relation has been thought to exist between Helicobacter pylori infection and gastric cancer. The present study was carried out to find correlation between $\mathrm{H}$. pylori and gastric carcinogenesis.

Methods: A case control study was performed in the department of endoscopy, Tribhuvan University Teaching Hospital, between January 2008 and February 2009. All patients having carcinoma stomach on endoscopic evaluation later confirmed by histopathological examination were included. Total 50 healthy individuals, with no positive finding in endoscopy were included in control group.

Results: Total 37 cases were analyzed. There were 23 (62.2\%) males and 14 (37.8\%) females. Majority were above 60 years $(46 \%)$. Blood group A was commonest group found in patients with carcinoma stomach. Distal part, antrum, 20 (54\%) was commonest location of tumor and Borrmann type three was commonest endoscopic type of tumor presentation. Majority of patients received triple therapy and $\mathrm{H}$. pylori detection was low in those treated with triple therapy. Distal tumors were significantly associated with increased prevalence of $\mathrm{H}$. pylori positivity. Total incidence of $\mathrm{H}$. pylori in cases was 54\% (20/37). In 15 (75\%) cases, H. pylori could be detected by both ELISA and biopsy method. Incidence of $\mathrm{H}$. pylori in controls was $64 \%$ (32/50). There was no statistically significant difference in H. pylori presence in cases and control.

Conclusions: Our study couldn't establish association between H. pylori and gastric cancer. More prospective trials can help find out correlation between combination of risk factors and gastric cancer.

Keywords: helicobacter pylori; gastric cancer; risk factors.

\section{INTRODUCTION}

Gastric cancer remains one of the common malignancies in many parts of the globe. ${ }^{1}$ Marshall and Warren were awarded the 2005 Nobel Prize in part of their discovery of $\mathrm{H}$. pylori and its causative role in gastric cancer The issue of carcinogenesis is yet unresolved. ${ }^{3}$ The etiopathological background may be different among people of this region. ${ }^{4} \mathrm{H}$. pylori is one of the important causes of gastric carcinoma. But there is still lack of final conclusion and the definite mechanism of their

Correspondence: Dr. Brindeswari Kafle, Gastroenterology Unit, Department of Medicine, Tribhuvan University Teaching Hospital, Kathmandu, Nepal. Email: drbindi20@gmail.com. 
association. Many researchers have done great amount of studies to reveal the relationship and the mechanism of the association of $\mathrm{H}$. pylori with gastric carcinoma. Most of them agree to the opinion that $\mathrm{H}$. pylori is a risk factor for gastric carcinoma, but a certain number of them hold different points of view. ${ }^{5}$ Helicobacter infection prevalence increases with age, but differs quite dramatically among populations. ${ }^{6} \mathrm{H}$. pylori gastritis has been associated with gastric carcinogenesis. ${ }^{7}$ $\mathrm{H}$. pylori is classified as a class I carcinogen by the World Health Organization. ${ }^{8} \mathrm{H}$. pylori strains with cagA gene are strongly associated with an increased risk of gastric adenocarcinoma. Recent studies suggest that the severity of gastric damage and eventual clinical outcome of $\mathrm{H}$. pylori infection are determined by $\mathrm{a}$ combination of host genetic and bacterial virulence factors. ${ }^{9}$ The grade of gastric atrophy (and therefore gastric cancer risk) is higher in patients with East Asian cag $A$ positive strains than in those with cagA-negative or Western cagA -positive strains. Of interest is that atrophy grade varies even among patients with East Asian cagA-positive strains, ${ }^{10}$ and that most $\mathrm{H}$. pyloriinfected subjects in fact develop no significant disease, remaining asymptomatic. The reasons for this are not explained by bacterial virulence factors alone; rather, genetic factors of the host should also be considered to play a role in $\mathrm{H}$. pylori induced outcomes. Khanna et al in India reported a lower prevalence of $\mathrm{H}$. pylori infection in gastric cancer patients than in the healthy controls, and suggested that $\mathrm{H}$. pylori may not be responsible for gastric cancer. ${ }^{4}$ On the other hand, Enroth et al showed that relative risk estimates for the association between $\mathrm{H}$. pylori and gastric cancer risk are to some extent determined by the diagnostic method used to detect $\mathrm{H}$. pylori infection. ${ }^{11}$ While some studies have reported no association with gastric cancer, the summary statistic derived from a meta-analysis indicated no doubt as to the existence of an association. ${ }^{12}$

\section{METHODS}

A prospective analytical study was performed in the department of endoscopy, Tribhuvan University Teaching Hospital, Kathmandu, Nepal, between January 2008 and February 2009 over fourteen months period. The total numbers of cases included and studied were 37 . Total of 50 age matched healthy individuals were included as control. Approval for the study was obtained from the department of surgery, department of medicine, TUTH, and Institutional Review Board of Institute of Medicine. For cases, all patients who were referred to endoscopy room and had carcinoma stomach on endoscopic evaluation later confirmed by histopathological examination were included in the study. Informed consent was obtained from all patients. The total numbers of patients included and studied were
37. Fifty healthy individuals, age matched with cases included in the study, presenting in endoscopy room for routine UGI endoscopy without any positive finding were included in the study as a control after taking consent. For cases, both sexes, any age undergoing upper Gl endoscopy for carcinoma stomach and giving consent to be enrolled in the study were included. Similarly for control group, both sexes giving consent, age matched with cases and undergoing routine upper GI endoscopy were included. Cases were excluded when there was inadequate endoscopic evaluation of the lesions with negative tissue biopsy. Any positive finding in endoscopy in control group was considered as exclusion criteria for controls. Trained senior gastrointestinal endoscopist performed all the endoscopies in the study group.

Patients and controls fulfilling the selection criteria were included in the study. Pre-procedure thorough history, physical examination and investigations were reviewed and a proforma was filled. Patients referred to endoscopy from inside or referred from other hospitals, both were included in the study if criteria were fulfilled according to cases or control selection. All the patients for endoscopy were advised to come with overnight fasting and patients were explained about the procedure in detail. Before the procedure pharyngeal anesthesia was given by $4 \%$ xylocaine spray. Intravenous hyoscine butyl bromide (Buscopan) was used when there were excessive stomach contractions making the procedure difficult. All the upper gastrointestinal endoscopies were done with standard technique following the principles of universal precaution using Olympus videoendoscope. All the endoscopies were performed by very experienced endoscopist. Thorough evaluation of the esophagus, stomach and duodenum was done. Lesion was identified. Location, extension and type of growth according to Borrmann classification was noticed and recorded. Multiple biopsies, at least six, were taken from the tumor margin for histopathological examination. Similarly, biopsy from tumor margin was also sent for histopathological examination for $\mathrm{H}$. pylori. Biopsy for $H$. pylori detection was also sent from antrum, body and fundus. Following the procedure, patients were advised to remain nil by mouth for an hour just to avoid any possible discomfort. Blood sample from the case was also collected and sent for serological diagnosis of H. pylori IgG by ELISA method. All the patients were advised to come for follow up with all the reports in the outpatient department, medical or surgical if patient were not admitted. In admitted cases, reports were traced in the wards. Bill numbers of the test given were also recorded to follow the reports in case the patients lost follow up. Details of the reports were collected and proforma was filled. In the control group similar principle was followed for performing UGI endoscopy. 
Biopsy for $\mathrm{H}$. pylori was taken from antrum, body and fundus and blood sample was taken for $\mathrm{H}$. pylori IgG detection by ELISA method.

Statistical analysis of the data was done using SPSS 13.0 (statistical package for social sciences). Association between carcinoma stomach and presence of H. pylori was done by Pearson's chi-square test. Proportion $(z)$ and correlation coefficient $(r)$ tests were done when and where applicable. The $p$-value of less than 0.05 was regarded as significant.

\section{RESULTS}

A total of 37 patients were included in the study and analyzed. Initially 42 patients were included for the study. But there was inadequate data, and follow up reports in five cases and so were excluded from the study. Finally 37 cases fulfilled all the criteria and were analyzed. Among 37 cases there were 23 (62.2\%) males and $14(37.8 \%)$ females with a ratio of $1.6: 1$ suggesting that males were having higher incidence of carcinoma stomach in our study. Majority of cases were above 60 years $(17 / 37,46 \%)$. Total 11 (30\%) cases were below 40 years, which showed that carcinoma stomach has rising incidence in younger age groups in our part. Some correlation has been shown between carcinoma stomach and blood group. We also assessed the type of blood groups (Table 1 ).

\begin{tabular}{|lll|}
\hline \multicolumn{2}{|l|}{ Table 1. Demographics. } & \\
\hline Parameter & Number & Percentage \\
Age distribution in yrs & & \\
$<40$ & 11 & $30 \%$ \\
$41-60$ & 9 & $24 \%$ \\
$>60$ & 17 & $46 \%$ \\
Blood group distribution. & \\
O & 16 & $43 \%$ \\
A & 18 & $49 \%$ \\
B & 2 & $5 \%$ \\
AB & 1 & $3 \%$ \\
Hemoglobin at & & \\
presentation & & \\
$\leq 7$ & 10 & $27 \%$ \\
$8-10$ & 18 & $49 \%$ \\
$>10$ & 9 & $24 \%$ \\
Alcohol/Smoking & & \\
Both & 21 & $58 \%$ \\
Alcohol or smoking & 4 & $11 \%$ \\
None & 12 & $12 \%$ \\
\hline
\end{tabular}

In all cases included in the study and it was found that commonest blood group was A type. Majority of the patients with carcinoma stomach present with history of malena and sometimes episodes of hematemesis developing anemia requiring blood transfusion. We also checked blood haemoglobin $(\mathrm{Hb})$ level (Table 1 ) in all the cases. Majority of patients were anemic with $\mathrm{Hb}$ level $10 \mathrm{gm} / \mathrm{dl}$ or below. Minimum $\mathrm{Hb}$ was $4 \mathrm{gm} / \mathrm{dl}$. Many studies have suggested that smoking and alcohol are risk factors for developing carcinoma stomach. We also evaluated incidence of smoking and alcohol in our patients of carcinoma stomach. We found that 21 (58\%) cases were consuming both alcohol and smoking thus increasing risk of carcinoma stomach. Total 12 (32\%) cases didn't consume alcohol or smoking. Many studies suggest that carcinoma stomach is found commonly in the distal part although incidence of proximal gastric cancer is also increasing. We also analyzed location of the tumor according to endoscopic finding. Our study showed that majority of the tumor was located in the distal stomach $(20 / 37,54 \%)$, followed by mid part (15, $40.5 \%$ ) and only two cases were found in proximal stomach.

Carcinoma stomach was also classified according to Borrmann endoscopic classification done for advanced carcinoma stomach. It was found that Type 3 (15, $40.5 \%$ ) was the commonest type of tumor morphology. Majority of patients before being diagnosed to have carcinoma stomach are initially treated with triple therapy regime for $\mathrm{H}$. pylori as they have vague symptoms of gastritis. We also analyzed whether the patients had received triple therapy in the past (Table 2). We found that 17 out of 37 (46\%) had received triple therapy. And it was found that majority of the patients who had received the therapy had $\mathrm{H}$. pylori negative by any methods. Some studies have shown that $\mathrm{H}$. pylori is associated more commonly with distal carcinoma stomach. We also assessed the association between tumor site and $\mathrm{H}$. pylori positivity. We found that the distal tumor was significantly associated with $H$. pylori infection (Table 3 ). Studies have also shown that $H$. pylori is more commonly a risk factor for developing carcinoma stomach in people. We also tried to correlate $\mathrm{H}$. pylori infection as a risk for developing carcinoma stomach according to age. But in our study, we found that $\mathrm{H}$. pylori was not significantly associated with carcinoma stomach in both the age groups above or below 45 years of age (Table 4). We evaluated the prevalence of $\mathrm{H}$. pylori infection in our patient with carcinoma stomach by histopathological examination and serum IgG detection by ELISA method. As shown (Table 5 ), in total $40 \%$ of cases, $\mathrm{H}$. pylori was detected by both method of diagnosis used in our study. Overall prevalence of $\mathrm{H}$. pylori in cases with carcinoma stomach 
Kafle et al. Association between Helicobacter Pylori and Gastric Cancer

was $54 \%$. Site specific presence of $\mathrm{H}$. pylori was also assessed on the basis of histopathological finding. We found that tumor combined with antral biopsy had more frequently positive histopathological test for $\mathrm{H}$. pylori (Table 5). Subjects in the control group were also analyzed. Total 50 controls were selected, age matched with the cases of carcinoma stomach. Details of the age distribution and $\mathrm{H}$. pylori positivity is shown in table 6 . Overall presence of $\mathrm{H}$. pylori detected by any of the diagnostic method in the study was $64 \%$ (Table 7). Presence of $\mathrm{H}$. pylori in cases and control were statistically analyzed. We found the difference was not statistically significant as shown in the table.

\begin{tabular}{|lll|}
\hline \multicolumn{2}{|l|}{ Table 2. Triple therapy and H. pylori. } \\
\hline Triple Therapy & H. pylori Positive & $\begin{array}{l}\text { H. pylori } \\
\text { Negative }\end{array}$ \\
Yes & $5(29 \%)$ & $12(71 \%)$ \\
No & $15(75 \%)$ & $5(25 \%)$ \\
\hline
\end{tabular}

Table 3. Tumor location and $\mathbf{H}$. pylori.

\begin{tabular}{|lll|}
\hline Tumor location & H. pylori Positive & P-value \\
$\begin{array}{l}\text { Antrum } \\
\begin{array}{l}\text { Others( mid, } \\
\text { proximal) }\end{array}\end{array}$ & $6 / 17(35 \%)$ & $<0.05^{*}$ \\
\hline
\end{tabular}

* Statistically significant by Chi-square test.

\begin{tabular}{|lll|}
\hline Table 4. Age group and H Pylori & \\
\hline Age group & H. pylori positive & P-value \\
$<45 y r s$ & $8 / 15(53 \%)$ & $>0.05 \% *$ \\
$\geq 45 y r s$ & $12 / 22(56 \%)$ & \\
\hline
\end{tabular}

* Statistically insignificant by Chi-square test

Table 5. Detection method, biopsy location and $\mathbf{H}$. pylori .

$\begin{array}{|lll|}\begin{array}{l}\text { Parameter } \\ \text { Detection method }\end{array} & \text { Number } & \text { Percentage } \\ \text { Both } & 15 & 40 \% \\ \text { Biopsy only } & 3 & 8 \% \\ \text { Elisa only } & 2 & 5 \% \\ \text { Total } & 20 & 54 \% \\ \text { Biopsy location } & & \end{array}$

\begin{tabular}{|lll|} 
Tumor only & 2 & $5 \%$ \\
Tumor and antrum & 12 & $12 \%$ \\
Antrum & 3 & $3 \%$ \\
Tumor and fundus & 1 & $1 \%$ \\
No H Pylori & 19 & $51 \%$ \\
\hline
\end{tabular}

Table 6. Prevalence of $\mathbf{H}$. pylori in controls.

\begin{tabular}{|lll|}
\hline Age groups & Number & H. pylori positivity \\
$<40 y r s$ & $20(40 \%)$ & $14 / 20(70 \%)$ \\
$41-60 y r s$ & $10(20 \%)$ & $7 / 10(70 \%)$ \\
$>60 y r s$ & $20(40 \%)$ & $11 / 20(55 \%)$ \\
Total & 50 & $32 / 50(64 \%)$ \\
\hline
\end{tabular}

Table 7. Association between cases and control.

\begin{tabular}{|lll|}
\hline $\begin{array}{l}\text { Cases(H. pylori } \\
\text { positive) }\end{array}$ & $\begin{array}{l}\text { Control } \\
\text { (H. pylori } \\
\text { positive }\end{array}$ & P-value (z-test) \\
$20 / 37(54 \%)$ & $32 / 50(64 \%)$ & $\begin{array}{l}>0.05 \text { (not } \\
\text { significant) }\end{array}$ \\
\hline
\end{tabular}

\section{DISCUSSION}

In our study we found that majority of our patients $(46 \%)$ were above 60 years of age suggesting increasing incidence of carcinoma stomach in older age groups. Total $30 \%$ of our cases were below 40 years of age. This shows that carcinoma stomach is increasing even in younger age groups. Study done at Memorial Sloan Kettering Cancer centre in 1600 cases of carcinoma stomach showed that carcinoma stomach was predominant after sixth decade but possibility of cancer remains at any age groups. ${ }^{13}$ Our study showed male female ratio of $1.6: 1$, which is similar to other studies showing ratio of 1.5:1 and suggesting higher incidence in males. ${ }^{14} \mathrm{We}$ also analyzed type of blood group in our cases with carcinoma stomach. Blood group A was the commonest (49\%) type found among cases. A study published in International journal of epidemiology in 2000 showed some association between carcinoma stomach and blood group $A$ suggesting some genetic predisposition in patients with blood group $A$ for development of carcinoma stomach. ${ }^{15}$ Total $25(68 \%)$ of cases had history of either long term 
smoking or alcohol or both. The relationship between stomach cancer and smoking has long been recognized. The European prospective investigation into cancer and nutrition project found a significant association between cigarette smoking, alcohol and gastric cancer. ${ }^{16}$ Gastrointestinal bleeding is usually occult and only occasionally massive in cases with carcinoma stomach. Anaemia and hematemesis are usually symptoms corresponding to advanced stage of disease. ${ }^{17}$ Majority of our patients in our part present at advanced stage and most of them have anaemia at presentation. In our study also, we found that more than $75 \%$ of cases had haemoglobin $<10 \mathrm{gm} \%$ suggesting chronic blood loss from the lesion.

Location of the tumor was also analyzed in our study. We found that distal (antrum) was commonest location of tumor suggesting distal part carcinoma stomach more frequent in our set up. In our study $54 \%$ cases were distal while body and proximal were $40 \%$ and $5 \%$ respectively. Proximal tumors were less frequent. Study done by Khanna et al in India in India also showed distal part $(66 \%)$ as common location of carcinoma stomach in this regions. ${ }^{4} \mathrm{H}$. pylori was positive in $70 \%(14 / 20)$ tumors located in the antrum while only in $35 \%(6 / 17)$ cases of carcinoma stomach in the body and proximal tumors. H. pylori has been associated with location of tumor as tumor of antrum and body has been associated with $\mathrm{H}$. pylori while cardiac tumors have not been found related. ${ }^{2}$ But in contrast to this, Khanna et al in India found $50 \%$ of cases of the cardiac region associated with $\mathrm{H}$. pylori. ${ }^{4}$ In our study. we had only two cases of proximal tumors and we could not comment on the association of proximal tumors with $\mathrm{H}$. pylori. When analyzing the treatment history, having completed triple regime against $\mathrm{H}$. pylori in recent past, we found that only $29 \%$ of cases that had received complete triple therapy in recent past were positive for $\mathrm{H}$. pylori. This suggests that complete eradication of $\mathrm{H}$. pylori is possible with complete course of triple therapy. ${ }^{18} \mathrm{We}$ also assessed the age group of carcinoma stomach with $\mathrm{H}$. pylori positivity. There was no statistically significant difference between in $\mathrm{H}$. pylori positivity both below and above 45 years of age. Khanna et al showed slightly higher $\mathrm{H}$. pylori positivity in younger patients. ${ }^{4}$ Similar other studies have also suggested that younger patient with $\mathrm{H}$. pylori are at higher risk of developing carcinoma stomach. ${ }^{19}$ In our study, we utilized histopathological examination (HPE) of biopsy tissue and ELISA for antibody detection for diagnosis of $\mathrm{H}$. pylori infection. We found that out of 20 positive cases, 15 were detected by both HPE and ELISA technique while three and two cases were diagnosed by biopsy only and ELISA only respectively. Blood antibody test sensitivity ranges from $76-84 \%$. The most reliable method for detecting $\mathrm{H}$. pylori infection is with endoscopic biopsy and histopathological examination, urease test or culture. ${ }^{20}$ Biopsies were taken from different sites during endoscopy in our study. It included biopsy from antrum, tumor and fundus. We found that highest possibility of getting $\mathrm{H}$. pylori was from tumor combined with antral biopsy. It may be explained by the fact that distal tumors are more frequent in our part. Although we couldn't prove it statistically, it can also be presumed that $\mathrm{H}$. pylori is a risk factor for developing distal tumors. Different studies done have also shown that $\mathrm{H}$. pylori is more frequently associated with distal tumors. ${ }^{21}$ In our study prevalence of $\mathrm{H}$. pylori in controls was $64 \%$. In developing countries the prevalence of $\mathrm{H}$. pylori is as high as $90 \% .{ }^{22}$ Actual infection rates vary from nation to nation. Developing nations have very high rates while in the west the prevalence rate is around $25 \%$, which is very low as compared to the developing countries. It has also been found that as age increases the prevalence rate of $\mathrm{H}$. pylori also increases. ${ }^{23}$ Prevalence rate of $\mathrm{H}$. pylori in control group and cases of carcinoma stomach were compared. In our study, prevalence rate was $64 \%$ in control and $54 \%$ in the cases. The difference was not statistically significant. It showed that the prevalence rate was higher in age matched controls. The similar study done in India showed $66 \%$ positivity in cases and $74 \%$ in control suggesting higher incidence in the age matched healthy controls. ${ }^{4}$ In another study done in Bangladesh by $M A$ Wajid et al showed $58 \%$ positivity in cases which was not statistically significant to suggest the possibility of $\mathrm{H}$. pylori as a risk factor for carcinoma stomach. ${ }^{24}$ From the results, we find that our study is not in concordance with the fact that $\mathrm{H}$. pylori is associated with increased risk of carcinoma stomach.

H. pylori has been etiologically linked to gastric cancer. $H$. pylori infection is more frequent in less developed Asian countries and is acquired at an early age than in more developed Asian countries like Japan and China. ${ }^{25}$ Frequency of gastric cancer, however, is very low in South Asian countries compared to that in Japan and China. Similar enigma has been reported from Africa as compared to the West. ${ }^{26}$ Seroprevalence of $\mathrm{H}$. pylori infection in adult populations of South Asian countries varies from $55 \%$ to $92 \%$. In contrast, seroprevalence of $\mathrm{H}$. pylori in Chinese and Japanese adults is $44 \%$ and $55 \%$, respectively. Annual incidence rate of gastric cancer in India, Bangladesh, and Thailand is 10.6, $1.3,7.1$ per 100000 populations, respectively; in contrast, that in China and Japan is 32-59 and 80115 per 100000 populations, respectively. ${ }^{26}$ Several studies from India failed to show higher frequency of $H$. pylori infection in patients with gastric cancer than controls. Available evidences did not support difference in $H$. pylori strains as an explanation for this enigma. Actual incidence of gastric cancer in Nepal has not been 
reported. One study done by $M L$ Shrestha et al in TUTH showed gastric cancer being one of the commonest malignancies in TUTH. ${ }^{27}$ But no studies have yet been done to establish an association between gastric cancer and $\mathrm{H}$. pylori. Despite established etiological role of $\mathrm{H}$. pylori, situation is somewhat different in Asian countries because in countries with higher frequency of infection, there is lower rate of gastric cancer. Host's genetic make-up and dietary and environmental factors might explain this enigma. This might be the similar reason in our set up for not being able to demonstrate significant association between carcinoma stomach and $\mathrm{H}$. pylori. Further studies are required to solve this issue.

\section{CONCLUSIONS}

In conclusion, our study couldn't establish correlation between $\mathrm{H}$. pylori and gastric carcinoma. Correlating our study and analyzing literature review, it seems that $\mathrm{H}$. pylori alone might not be the only independent factor in gastric carcinogenesis. Hosts genetic factor and dietary factors may play a major role in determining whether a patient infected with $\mathrm{H}$. pylori will develop gastric carcinoma. There may be other factors acting together with $\mathrm{H}$. pylori to cause gastric carcinoma. More prospective studies are recommended to establish association between combination of risk factors and gastric cancer.

\section{REFERENCES}

1. Ando T, Goto Y, Ishiguro K, Maedo O, Watnabe O, Ohmiya N, et al. The interaction of host genetic factors and Helicobacter pylori infection. Inflammopharmacology. 2007;15(1):10-4.

2. Tang YL, Gan RL, Dong BH, Jiang RC, Tang RJ. Detection and location of Helicobacter pylori in human gastric carcinomas. World J Gastroenterol. 2005 Mar 7;11(9):1387-91.

3. Sipponen P, Kosunen TU, Valle J, Rihela M, Seppala K. Helicobacter pylori infection and chronic gastritis in gastric cancer. J Clin Pathol. 1992; 45:319-23.

4. Khanna AK, Seth P, Nath G, Dixit VK, Kumar M. Correlation of Helicobacter pylori and gastric carcinoma. J Postgrad Med. 2002;48:27-8.

5. . Zhuang XQ, Lin Sr. Progress in research on the relationship between H Pylori and gastric cancer. Shijie Huaren Xiaohuq Zazhi. 2000;8:206-7.

6. Dooley CP, Cohen H, Fitzgibbons PL, Bauer M, Appleman MD, Perez- Perez GI, et al. Prevalence of Helicobacter pylori infection and histologic gastritis in asymptomatic persons. $\mathrm{N}$ Engl J Med. 1989;321:1562-6.

7. Plummer M, Franceschi S, Munoz N. Epidemiology of gastric cancer. IARC Sci Publ. 2004;157:311-26.

8. Xue FB, Xu YY, Wan Y, Pan BR, ren J, Fan DM. Association of $\mathrm{H}$ pylori infection with gastric carcinoma: a Meta analysis. World J Gastroenterol. 2001;7(6):801-4.

9. El-Omar EM, Carrington $\mathrm{M}$, Chow $\mathrm{WH}$, McColl KE, Bream JH, Young HA, et al. Interleukin-1 polymorphisms associated with increased risk of gastric cancer. Nature. 2000;404:398-402.

10. Azuma T, Yamazaki S, Yamakawa A. Association between diversity in the Src homology 2 domain-containing tyrosine phosphatase binding site of $\mathrm{H}$ pylori CagA protein and gastric atrophy and cancer. J Infect Dis. 2004;189:820-7.

11. Enroth H, Kraaz W, Rohan T, Nyren O, Engstrand L. Does the method of $\mathrm{H}$ pylori detection influence the association with gastric cancer risk? Scand J Gastroenterol. 2002;37:884-90.
12. Eslick GD, Lim LL, Byles JE, Xia HH, Talley NJ. Association of $\mathrm{H}$ pylori infection with gastric carcinoma: a meta-analysis. Am J Gastroenterol. 1999;94:2373-9.

13. Brennan MF. Gastric adenocarcinoma. In: Zinner MJ, Ashley SW, editors. Maingot's abdominal operations. 11th ed. New York: McGraw-Hill; 2007. p. 417-7.

14. Ferlay J, Bray F, Pisani P, Parkin DM. GLOBOCAN 2002 cancer incidence, mortality and prevalence worldwide. IARC cancerbase no.5. version 2.0. Lyon: IARC press; 2004.

15. You WC, Ma JL, Liu W, Gail MH, Chang YS, Zhang L, et al. Blood type and family cancer history in relation to precancerous gastric lesions. Int J Epidemiol. 2000 Jun;29(3):405-7.

16. Maguire A, Porta M, Sanz-Anquela JM, Ruano I, Malats N, Piñol JL. Sex as a prognostic factor in gastric cancer. Eur J Cancer. 1996 Jul;32A(8):1303-9.

17. Catalano V, Labianca R, Beretta GD, Gatta G, de Braud F, Van Cutsem E. Gastric cancer. Critical Reviews in Oncology/ Hematology. 2009 Aug;71(2):127-64.

18. Mirbagheri SA, Hasibi M, Abouzari M, Rashidi A. Triple, standard quadruple and ampicillin-sulbactam-based quadruple therapies for $\mathrm{H}$. pylori eradication: a comparative three-armed randomized clinical trial. World J Gastroenterol. 2006 Aug 14;12(30):4888-91.

19. Liu Y, Kaneko S, Sobue T. Trends in reported incidences of gastric cancer by tumour location, from 1975 to 1989 in Japan. Int J Epidemiol. 2004;33:808-15.

20. Logan RP, Walker MM. ABC of the upper gastrointestinal tract: Epidemiology and diagnosis of Helicobacter pylori infection. BMJ. 2001;323:920-2.

21. Whiting JL, Hallissey MT, Fielding JW, Dunn J. Screening for gastric cancer by Helicobacter pylori serology: a retrospective study. Br J Surg. 1998;85:408-11.

22. Crespi M, Citarda F. Helicobacter pylori and gastric cancer: what is the real the real risk? Gastroenterologist. 1998;6:16-20. 
23. Pounder RE, Ng D. The prevalence of Helicobacter pylori infection in different countries. Aliment Pharmacol Ther. 1995;9:33-9.

24. Majid MA, Faruq TIMA, Hossain ABMB. Association of Helicobacter pylori infection with gastric carcinoma. Bangladesh Med Res Counc Bull. 2009;35:7-10.
25. Miwa H, Go MF, Sato N. H pylori and gastric cancer: the Asian enigma. Am J Gastroenterol. 2002;97(5):1106-12.

26. Singh K, Ghoshal UC. Causal role of Helicobacter pylori infection in gastric cancer: an Asian enigma. World J Gastroenterol. 2006 Mar 7;12(9):1346-51.

27. Shrestha ML. Gastric cancer: diagnosis and treatment options. JIOM. 2006;28(3):43. 